\title{
Quality Improvement in Neonatal Intensive Care Units
}

\section{Eun Sun Kim}

Department of Pediatrics, Kangwon National University School of Medicine, Chuncheon, Korea

\section{ABSTRACT}

Even with the increasing number of high risk infants, neonatal care in Korea has undergone development with improved survival rate. This rapid improvement in the outcomes brought care quality in neonatal intensive care unit (NICU) to the surface. Quality improvement (QI) involves safe, timely, effective, efficient, equitable, and patient-centered care. In this review, methods of QI are described with examples of NICU QI topics. Each NICU can voluntarily develop a QI project, but systematic supports are essential. As human and systemic resources in NICUs in Korea are insufficient, institutional and national supports are necessary to attain QI. Furthermore, collaborative neonatal network can provide a QI standard and evidence based-medicine, as well as QI research.

Key Words: Quality improvement, Neonatal intensive care unit, Network

서론

우리나라 통계청 자료에 따르면 ${ }^{1)}$, 국내 총 출생아는 1993년 715,826명에서 2016년 406,243명 으로 23 년간 약 $44 \%$ 정도의 급격한 감소를 보여 저 출산이 큰 문제가 되고 있다. 그러나 출생 체 중 2,500g 이하의 저체중출생아(low birth weight infant, LBWI)는 1993년 18,532명에서 2016년 에는 23,829 명으로 총 출생아의 $2.6 \%$ 에서 $5.9 \%$ 로 비율이 늘어났고, 출생 체중 $1,500 \mathrm{~g}$ 이하의 극 소저체중출생아(very low birth weight infant, VLBWI)는 1993년 929명에서 2016년에는 2,783명 으로 총 출생아의 $0.13 \%$ 에서 $0.69 \%$ 로 증가하여 수적으로는 $530 \%$ 의 증가를 보였다. 저체중아와 미숙아의 증가는 산모의 고령화와 불임의 증가, 그리고 이에 따른 인공 임신의 증가로 조산아와 다태아 출생이 증가되는 것에 그 원인이 있다.

고위험 신생아의 비율이 증가함에 따라 신생아중환자실(neonatal intensive care unit, NICU) 에서의 양질의 치료가 중요해지고 있다. 국내에서는 학술 연구 용업 사업의 하나로 한국 신생아 네트워크(Korean Neonatal Network, KNN)가 2013년 4월에 출범하게 되었고 ${ }^{2)}$, 신생아 의사들의 주도로 전국 NICU의 VLBWI 등록 사업을 진행하고 있다. 이 사업을 통해 보았을 때 국내의 신생 아 집중 치료술은 최근 상당한 발달을 이루어 VLBWI의 생존율이 현저히 향상되었지만 각 NICU 별 생존율 및 합병증의 발생 빈도는 편차를 보이고 있다. KNN에서는 2017년에 신생아 패혈증과 중심정맥관 관련 혈류감염(central line associated blood stream infection, CLABSI)률을 시작으 로 질 향상(Quality improvement, QI) 활동을 시작하였고, 벤치마킹 병원과 QI 활동 병원을 대상 으로 2년간 공식적인 QI 활동을 진행할 예정에 있다.

"모든 의료진은 매일 직장에서 두 개의 업무를 하게 되는데, 하나는 직장 일 자체이고, 다른 하
Received: 9 May 2018

Revised: 17 May 2018

Accepted: 23 May 2018

Correspondence to: Eun Sun Kim

Department of Pediatrics, Kangwon National University Hospital, Kangwon National University School of Medicine, 156 Baengnyeong-ro, Chuncheon 24289, Korea

Tel: +82-32-258-2022

Fax: +82-32-258-2418

E-mail: naivesun1@hanmail.net

Copyright(c)

By Korean Society of Neonatology.

All right reserved.

This is an Open-Access article distributed under the terms of the Creative Commons Attribution Non-Commercial License (http://creativecommons.org/licenses/ by-nc/4.0), which permits unrestricted non-commercial use, distribution, and reproduction in any medium, provided the original work is properly cited. 
나는 그 일을 향상시키는 일이다"라고 미국의 QI 전문가 Batalden과 Davidoff $^{3)}$ 은 언급했었고, 이는 QI의 속성을 가장 잘 표현하고 있는 말이다. QI의 필요성을 일찍이 인지하고 정책으로서 자리잡은 미국 은 2000년대 초반에 이미 3-5년 내에 약 10억 달러 정도의 “의료 질 혁신 자금" 확보가 필요하다고 애기하며 정부에서도 의료 질 관리에 관여하고 의료기관 내부에서도 책임감을 갖고 의료 질 관리에 적극 적일 수 있는 분위기를 조성하였단)

국내에서는 아직 정부 지원 등을 통한 QI가 자리잡지는 못하였으 나 의료진 자체의 요구도에 따라 병원 개개의 능력별로 QI를 진행 하고 있으며 KNN을 통해 네트워크 QI도 시작하는 단계이다. 이에 $\mathrm{NICU}$ 내에서의 QI의 의미와 실제 진행 방법에 대해 논의하고자 한다.

\section{$\mathrm{NICU}$ 에서의 질 향상이란?}

2000년대 초반 미국에서는 주요 질환을 않고 있는 성인들과 외래 진료를 받는 소아들이 적절한 시기에 치료를 받고 있는지 12 개 대도 시를 중심으로 조사를 했었고, 성인의 $54.9 \%$ 와 소아의 $46.5 \%$ 에서만 적절한 진료를 받고 있다는 충격적인 결과를 보여줬다 ${ }^{5,6)}$. 2000년대 초반부터 미국에서는 Institute of Medicine (IOM)을 중심으로 복잡 한 의료 체계에서 의료의 질 관리 방침을 위한 정책의 필요성을 공유 하며 공공부문과 사립부문에서 동시에 투자를 통해 조직적으로 의 료 질 관리를 해오고 있다,7).

미국의 IOM에서 정의한 바에 따르면, 의료의 질은 "바람직한 의 료 결과를 얻을 가능성이 많도록 그리고 현재의 의료 지식에 합당 한 의료 결과를 받도록 하기 위한 개개의 의료 서비스의 정도"이다 ${ }^{4,8)}$. 적절한 의료를 제공하려면 질 적인 측면에서 도달해야 할 6 가 지 영역이 있다. 의료는 환자에게 안전해야(safe)하고, 시기 적절 해야(timely)하며, 남용되지 않고 제대로 이용되도록 효과적으로 (effective) 전달되어야 하며, 의료 장비나 도구 등이 낭비되지 않게 효율적(efficient)이어야 하고, 환자에 따른 차별 없이(equitable) 환 자 중심으로(patient-centered) 이루어져야 한다 ${ }^{4,8)}$. 위의 영역의 앞 자를 따서 STEEEP이라고 간단히 명하기도 하는데, STEEEP을 추구 하는 의료가 제공된다면 환자는 좀 더 안전하고 믿을만하며 집약적 이고 유용한 의료 혜택을 받을 수 있을 것이다. 의료의 질을 평가하 는 지표로 Donabedian")은 (1) 구조(structure), (2) 과정(process), (3) 결과(outcomes)의 세 가지 영역을 제시하였다. (1) 구조는 시설, 장 비, 서비스, 치료 인력 등을 포함하며, NICU에서는 환자 당 간호인 력, 면적 당 환자 수, 격리실 개수, 인공호흡기 개수, 영상기계의 즉각 이용 가능성, 소생술이 가능한 인력 수 등이 해당된다. (2) 과정은 임 상에서 환자에게 전달되기까지의 활동의 전반을 포함하며, 예를 들 어 NICU에서는 환자 접촉 전 손 씻기 수행 정도, 호흡곤란증후군 치 료로 출생 후부터 인공계면활성제를 주기까지의 시간, 미숙아 망막
증 검사를 제 시간에 수행하는 비율, 미숙아 분만 전 산모 스테로이 드 투여 비율, 모유수유 비율 등이 해당된다. (3) 결과 지표는 NICU 내에서는 신생아 사망률, 패혈증 빈도, 기관지폐이형성증 빈도, 미숙 아 망막증 빈도, 뇌출혈 빈도, CLABSI 빈도 등을 예로 들 수 있다.

\section{질 향상의 방법}

질 향상 활동에 앞서 각 분야의 리더들의 모인 다학제 팀의 구성이 필요하다. 대개의 질 향상 활동은 그 일을 하고 있는 사람들 중 대표 성이 있는 사람들로 구성되며, 팀 리더는 모든 일들을 가장 잘 알고 있는 사람으로 선발하고, 팀의 조력자는 질 향상 활동 경험이 풍부하 고 개선활동 방법들을 잘 알고 있는 사람으로 구성한다. 그 외에 기 술적인 전문가는 중환자 의사, 일상 환자 관리의 리더는 관리자급 간 호사, 행정적인 리더는 병원 행정직이 되며, 임상 약사, 임상 간호 관 리자 등이 팀원으로 속할 수 있다.

질 향상 활동의 방법은 Care model, Lean model, Six Sigma, focusanalyze-develop-execute (FADE), total quality management (TQM), 그리고 plan, do, study, act (PDSA) cycle 등 다양한 방법으로 시행할 수 있으나, 의료계에서 일반적으로 시행하는 PDSA cycle을 소개하 면 다음과 같은 5단계의 과정을 거치게 된다(Figure 1). (1) 왜 향상 이 필요한 지 알고 있어야 한다(목적). (2) 향상이 일어나는지를 알 수 있는 피드백 기전을 가지고 있어야 한다(측정). (3) 향상을 가져 오기 위해서는 효과적인 변화 방법을 만들어내야 한다(변화). (4) 변 화를 정착시키기 전에 변화를 시험해 보아야 한다. (5) 언제, 어떻게 변화를 정착시킬 것인지 알고 확립한다 ${ }^{8,10)}$.

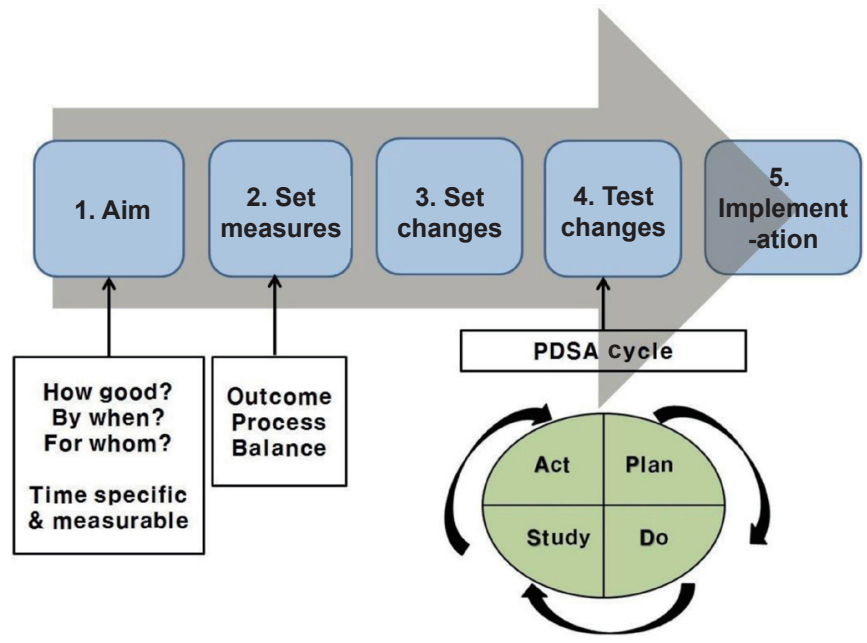

Figure 1. Methods of quality improvement. Abbreviation: PDSA, plan, do, study, act. 


\section{1. 목표 설정}

질 향상의 목표는 얼마나 좋게, 언제까지 할 것인지를 포함한다. 누구 혹은 어떤 시스템을 위해서인지도 생각해 볼 수 있다. 목표 설 정은 구체적이고(specific), 측정 가능하고(measurable), 달성 가능 해야 하고(attainable), 적절한 관련성이 있으며(relevant), 시기 적절 해야(timely) 한다(SMART) ${ }^{8,10)}$. NICU에서 생각해 볼 수 있는 목표의 예를 들어보자. "향후 6개월 이내에 NICU 퇴원 후 재입원율을 10\% 에서 5\%까지 줄이겠다”, 혹은 “2019년 3월까지 신생아실 퇴 원아의 완전 모유수유율을 50\%에서 80\%까지 올리겠다" 등을 생각해 볼 수 있겠다. 이러한 구체적인 목표 설정을 위해서는 목표로 하는 영역에 서 현재 자료의 수집이 필요하며 이는 향후 향상의 여부를 확인할 수 있는 기초자료가 된다.

\section{2. 측정 방법 확립}

변화의 시도 후에 항상 향상이 이루어지는 것은 아니기 때문에 시 도한 변화를 적용할 지, 도입해볼 지, 버릴지를 정하는 기준이 필요 하다. 이를 위해서 자료를 측정하는 방법이 확립되어야 한다. 목표 와 관련된 측정 방법들을 정하되 간단하고 실용적인 측정 방법이 효 율적이다. 측정된 자료들은 그래프화하여 결과를 보는 것이 유용하 다. 측정의 내용에 따라, (1) 결과 측정(outcome measure), (2) 과정 측정(process measure), (3) 균형 측정(balance measure)으로 나뉜 다 ${ }^{8,10)}$. 결과 측정은 최종적으로 바뀌었으면 하는 지표를 측정하는 것이고, 과정 측정은 결과를 바꾸기 위한 과정 수행의 단계나 부분을 측정하는 것이며, 균형 측정은 좋은 결과를 만들기 위한 변화가 다른 방면에서 부작용은 없었는지를 측정하는 것이다. 예를 들어, "2019 년 3월까지 신생아실 퇴원아의 완전 모유수유율을 50\%에서 80\%까 지 올리겠다"라는 목표와 관련하여, (1) 퇴원시의 완전 모유수유율 (\%), (2) 분만 후 첫 모유수유까지의 시간, (3) 간호사의 산모 모유수 유 교육 빈도, (4) 수유문제로 인한 재입원율 등의 측정 방법을 세웠 다면, (1)은 결과 측정, (2)와 (3)은 과정 측정, (4)는 균형 측정 방법 이 될 것이다. 측정 자료는 그래프화하여 시간에 따른 추이를 보는 것이 매우 유용하다 ${ }^{11)}$.

\section{3. 변화 설정}

모든 문제에는 잠재적인 원인과, 악화요인, 장애요소가 있기 때문 에 향상을 위한 변화의 설정 전에 현 상태의 변화를 발목 잡고 있는 원인을 먼저 분석하여야 한다. QI를 시작하고 변화가 있을 수도 있 으나 장기적으로 현 수준에 머무르는 변화는 반응적인 변화(reactive change)일 뿐이기 때문에 근본적인 변화(fundamental change)를 일으키기 위해서는 시스템을 전반적으로 혹은 부분적으로 바꿀 정 도의 변화가 필요할 수 있다. 이를 위해서는 QI 팀 내에서의 brainstorming이 필요하다. QI 주제와 관련된 근거 문헌을 조사하거나 잘 되고 있는 곳을 벤치마킹 하는 것도 방법이 될 수 있다. 그 외 변
화 설정의 접근법은 Fishbone diagram (cause-and-effect diagram), Driver diagram, Force field analysis, Process mapping 등이 있다. 이 중 근본 원인 분석(root cause analysis)에 좋은 방법의 예는 Fishbone diagram이다(Figure 2) ${ }^{8,10)}$.

\section{4. 변화 시험}

변화의 시도가 항상 향상으로 이어지는 것은 아니기 때문에 변 화는 시험대에 올라야 한다. 계획(plan)-실행(do)-분석(study)-조치 (act)의 PDSA cycle이 변화를 시험하는 방법이다(Figure 1). 계획단 계에서는 목표에 맞춰 누구에게 무엇을 언제 어떻게 할지 정하고 자 료 수합을 계획한다. 실행단계에서는 계획을 수행하면서 문제점이 나 예상치 못했던 사항들을 기록하고 자료를 분석하기 시작한다. 분 석단계에서는 자료 분석을 완성하여 자료를 비교하고 배울 점이 있 었는지 정리한다. 조치의 단계에서는 어떤 변화를 만들어야 하고 다 음 cycle은 어떻게 진행할지를 결정한다. PDSA cycle에서 실패의 경험을 해도 실패 자체가 변화를 위한 공부를 가속시키므로 PDSA cycle을 계속 돌려보는 것이 중요하다. 또한 한가지 목표와 관련하 여 여러 하위 목표가 있는 경우에는 여러 QI의 PDSA cycle을 동시에 시행할 수도 있다(Figure 3) ${ }^{8,10)}$.

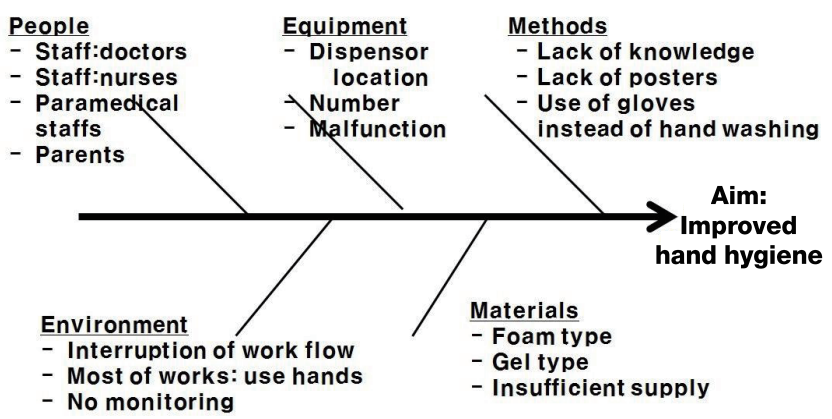

Figure 2. An example of fishbone diagram.

\section{Multiple PDSA cycle ladders for QI}

Aim: Reduce infection in NICU

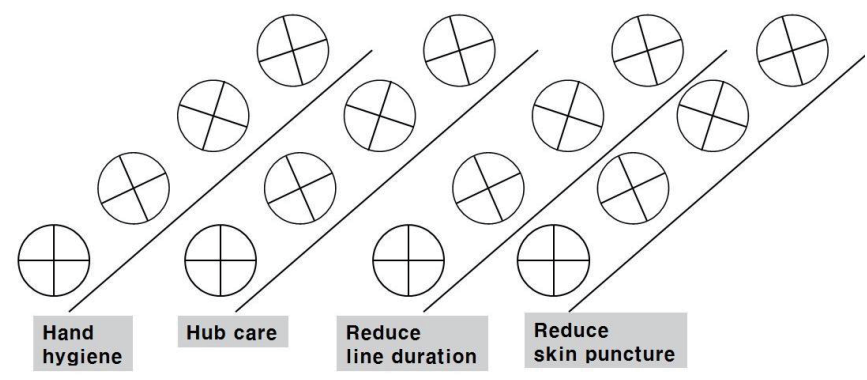

Figure 3. An example of multiple plan, do, study, act (PDSA) cycles. Abbreviations: NICU, neonatal intensive care unit; QI, quality improvement. 


\section{5. 변화 확립}

PDSA cycle을 통해 만들어진 변화를 시스템에 확립시키기 위해서 는 추가적인 노력이 필요하다. 변화의 표준화 작업 및 문서화 작업 이 필요하다. 일상의 근무자들이 변화에 익숙해지도록 교육이 필요 하고 교육의 정도를 측정하여 관리하고 유지하기 위한 지원을 확보 한다 ${ }^{8,10)}$.

\section{$\mathrm{NICU}$ QI의 나아갈 방향}

QI는 자발적인 활동이나 현 국내 NICU 환자들에게 양질의 의료 를 제공하려면 다학제 QI 팀을 구성하여 체계적으로 진행하는 것이 필요하다. QI를 처음 시행하는 경우에는 방법적인 면에 있어서 병원 의 지원이 필요할 수 있다. NICU QI를 진행하고 있는 미국의 한 대 형병원의 예를 들면, NICU 내 CLABSI율, 중심정맥관 Bundle 수행 률, 손 위생률, 비의도적 발관(Unplanned extubation)율, 말초혈관 주사 혈관외유출(peripheral intravascular catheter infiltration/extravasation)률, 병원에서 생긴 압박 손상(hospital acquired pressure injury) 빈도, 인공호흡기 관련 사건(ventilator associated events) 빈 도, 모유 혼선율, 안전 문제, 사망률, 퇴원 후 7 일이내 재입원율 등의 주제로 주기적으로 QI 회의를 통해 수행 향상을 도모한다. 각각의 주제에 대해 실무팀이 PDSA cycle을 시험해보며 자료 분석을 통해 변화의 나아갈 방향을 정한다. 국내 NICU는 위에 예로 든 미국의 대 형병원과 비교 시 다양한 QI를 진행하기에 경험과 인력 부족이 있을 것이므로 현재 해당 NICU에서 가장 문제가 되고 있는 사안에 대한 기초 자료 조사와 함께 QI를 시작해 볼 수 있다. 현재 국내 NICU 실 정에서는 의료인의 수, 장비, 환경 등 많은 부분에서의 시스템이 열 악하기 때문에 QI를 진행하는데 있어서 병원의 협조가 필요할 수 있 고, $\mathrm{QI}$ 를 하다가 발견되는 시스템의 개선을 위해서 병원의 지원이 필요할 수 있다. 국내의 NICU에서는 산발적으로 QI 활동이 있어왔 고 2016년부터 대한신생아학회 서울 · 경인지회에서 미니심포지엄 의 형태로 "NICI에서의 QI: 경진대회”가 진행되어왔다. 다른 NICU 에서 하고 있는 QI 활동을 참조하여 각 $\mathrm{NICU}$ 실정에 맞는 QI를 시행 해 볼 수 있을 것이다.

국내 NICU 개개의 QI 뿐만 아니라 네트워크를 통한 협력적인 QI 관리 또한 필요하다. Vermont Oxford Network (VON), Canadian Neonatal Network (CNN), California Perinatal Quality Care Collaborative (CPQCC) 등 외국의 신생아 협력 네트워크에서는 QI 관련 연 구를 수행하고 질병률에 대한 기본자료를 제공한다 ${ }^{12,13)}$. 또한 미 국의 American Academy of Pediatrics (AAP)에서도 'the Steering Committee on Quality Improvement and Management'를 운영해 오 며 증거기반 의료 및 $\mathrm{QI}$ 의 방법론에 대한 학문적 자료를 검증하며 제공해오고 있다 ${ }^{14,15)}$. 국내에서는 향후 $\mathrm{KNN}$ 을 통해 국내 $\mathrm{NICU}$ 의
QI 관련 연구 및 기초자료를 제공할 수 있을 것이다. 또한 외국의 신 생아 협력 네트워크의 자료와 비교하며 증거기반 의료에 바탕을 두 고 QI의 나아가야 할 방향도 설정할 수 있을 것이다. 국내의 네트워 크뿐만 아니라 국내 학회 차원에서도 QI 수행을 위한 교육 및 가이 드를 통해 각 $\mathrm{NICU}$ QI를 지원한다면 체계적인 NICU QI 확립에 도 움이 될 것이다.

\section{결론}

고위험 신생아의 증가로 NICU는 양적인 성장과 더불어 고도의 발 전을 해왔지만 이제는 질적인 성장도 뒤따라야 하는 시대가 왔다 ${ }^{16)}$. 국내에서는 $\mathrm{KNN}$ 을 중심으로 네트워크 $\mathrm{QI}$ 활동이 시작되었고 각 기 관에서도 자체적으로 QI를 하려는 노력들이 잇따르고 있다. NICU 내에서의 QI 활동은 다학제 팀을 구성하고 현재 우선시 되는 문제 들과 관련된 목표를 설정하여 PDSA cycle을 통해 진행해볼 수 있다. 리더는 문제점에 대해 개개인을 탓할 게 아니라 잘못된 시스템을 향 상시키도록 변화시켜야 함을 이해하고 필요 시 병원의 협조를 통한 시스템의 정착을 위해 노력해야 할 것이다. 각 NICU 및 각 기관의 노 력으로 QI를 유지하는 것에는 한계가 있을 수 있으므로 외부에서의 지원이 뒷받침 되어야 하며 개개 기관의 의료 질에 차이가 지속되는 부분에 대해서는 원인 분석을 통한 지원이 필요할 것이며 ${ }^{13)}$ 의료인 의 QI 수행 훈련도 지원되어야 할 것이다 ${ }^{17)}$.

\section{REFERENCES}

1) Korean Statistical Information Service. Birth statistics [Internet]. Daejeon: KOSIS; 2018 [cited 2018 May 21]. Available from: http://www.kosis.kr.

2) Chang YS, Park HY, Park WS. The Korean Neonatal Network: an overview. J Korean Med Sci 2015;30 Suppl 1:S3-11.

3) Batalden PB, Davidoff F. What is "quality improvement" and how can it transform healthcare? Qual Saf Health Care 2007; 16:2-3.

4) Institution of Medicine US, Committee on Quality of Health Care in America. Crossing the quality chasm: a new health system for the 21st century. Washington, DC: National Academy Press, 2001.

5) McGlynn EA, Asch SM, Adams J, Keesey J, Hicks J, DeCristofaro A, et al. The quality of health care delivered to adults in the United States. N Engl J Med 2003;348:2635-45.

6) Mangione-Smith R, DeCristofaro AH, Setodji CM, Keesey J, Klein DJ, Adams JL, et al. The quality of ambulatory care delivered to children in the United States. N Engl J Med 2007;357: 
1515-23.

7) Kohn LT, Corrigan J, Donaldson MS. To err is human: building a safer health system. Washington, DC: National Academy Press, 2000.

8) Langley G, Moen R, Nolan K, Nolan T, Norman C, Provost L. The improvement guide: a practical approach to enhancing organizational performance. 2nd ed. San Francisco: Jossey-Bass Press, 2009

9) Donabedian A. Explorations in quality assessment and monitoring. The definition of quality and approaches to its assessment. Vol. 1. Ann Arbor: Health Administration Press, 1980.

10) Institution for Healthcare Improvement. Open school [Internet]. Boston: Institution for Healthcare Improvement; c2018 [cited 2018 May 21]. Available from: http://www.ihi.org/ education/ihiopenschool.

11) Provost LP, Murray S. The health care data guide: learning from data for improvement. San Francisco: Jossey-Bass Press, 2011.
12) Chawla D, Suresh GK. Quality improvement in neonatal care: a new paradigm for developing countries. Indian J Pediatr 2014; 81:1367-72.

13) Balakrishnan M, Raghavan A, Suresh GK. Eliminating undesirable variation in neonatal practice: balancing standardization and customization. Clin Perinatol 2017;44:529-40.

14) American Academy of Pediatrics Steering Committee on Quality Improvement and Management. Classifying recommendations for clinical practice guidelines. Pediatrics 2004; 114:874-7.

15) Isaac A, Saginur M, Hartling L, Robinson JL. Quality of reporting and evidence in American Academy of Pediatrics guidelines. Pediatrics 2013;131:732-8.

16) Berwick DM. Era 3 for medicine and health care. JAMA 2016; 315:1329-30

17) Ehret DY, Patterson JK, Bose CL. Improving neonatal care: a global perspective. Clin Perinatol 2017;44:567-82. 